\title{
De Wilde, Pieter, Ruud Koopmans, Wolfgang Merkel, Oliver Strijbis und Michael Zürn, Hrsg. 2019. The Struggle Over Borders. Cosmopolitanism and Communitarianism
}

\section{Cambridge, London: Cambridge University Press. 276 Seiten, Hardback 59,99 £, Paperback 22,99 £, e-book $24 £$}

\section{Claus Leggewie}

Online publiziert: 29. Mai 2020

(C) Der/die Autor(en) 2019

\section{Neues zur K-Frage: Kosmopoliten und Kommunitäre in neuen Konfliktlinien}

David Goodharts (2017) griffige Gegenüberstellung von ,Somewheres', an ihrem Lebensmittelpunkt Verwurzelten, und ,Anywheres', die heute hier und morgen dort leben können, ist zum Gemeinplatz geworden. Sie dient nicht nur als Illustration unterschiedlicher Mobilitätsmuster, sondern wird auch normativ eingesetzt: Anywheres gelten oft als egozentrische Globalisierungsgewinner, die mit ihrer Arbeitsweise, ihrem Lebensstil und ihrer Weltanschauung den Rest der Welt abhängen. Unter vermeintlich „Abgehängten“ grassiert bisweilen Wut, wenn nicht Hass auf diese hypermobile Elite, sie glauben, ihnen seien mit der frivolen Entgrenzung dieer gemeinschaftlichen Grundlagen geraubt worden. Gegen die „Kosmopoliten“ (Weltbürger) halten sie Heimat und Herkunft hoch, und dieser Terminus hat sich auch unter welterfahrenen Zeitgenossen zur (selbst)kritischen Größe bei der Ursachenanalyse des elitenfeindlichen, protektionistischen, oftmals völkisch-autoritären Nationalismus entwickelt.

Ob es sich bei diesem „Kampf um die Grenzen“ um eine oberflächliche Zuschreibung oder eine tiefgehende Spaltungs- und Konfliktlinie in heutigen Gesellschaften handelt, will der profunde, zugleich neue Fragen aufwerfende Band klären, den Angehörige des Berliner Wissenschaftszentrums WZB (Ruud Koopmans, Wolfgang Merkel und Michael Zürn) mit Kollegen aus Trondheim und Zürich vorgelegt haben. Die eher intuitive Vermutung einer tektonischen Verschiebung der kulturellen, strukturellen und organisatorischen Grundlagen liberaler Gesellschaften (vgl. Leggewie 2017, 2019, S. 189 ff) wird derzeit in diversen Projekten ausgelotet. Die Autoren dieses Bandes, der Spezialisten wie Studierenden nachdrücklich empfohlen sei, fragen auf der Grundlage eines datengestützten Fünf-Länder-Vergleichs (USA und Mexiko

Prof. Dr. C. Leggewie $(\bowtie)$

Ludwig Börne-Professur, Zentrum für Medien und Interaktivität, Justus-Liebig-Universität Gießen, Ludwigstraße 34, 35390 Gießen, Deutschland

E-Mail: Claus.Leggewie@zmi.uni-giessen.de 
für die NATFA-Region, Deutschland, Polen und die Türkei für die EU-Zone) in Beiträgen von Strijbis und de Wilde et al. auch nach den inter- und transnationalen Dimensionen und Verwerfungen dieses Erdbebens. Ihr Anspruch ist, eine tragfähige Erklärung für die ubiquitäre Emergenz populistischer, gegen die ökonomische, kulturelle und politische Globalisierung gerichteter Bewegungen und Parteien zu liefern, die - das ist die Pointe - überkommene Rechts-Links-Schemata sprengen.

„Kosmopolitans“ und „Communitarians“ sind Begrifflichkeiten aus der politischen Philosophie, die Koopmans und Zürn im konzeptionellen Einleitungsbeitrag in die politische Soziologie übertragen. Sie rekurrieren auf das ,cleavage“-Theorem, das Seymour M. Lipset vor über fünfzig Jahren mit Stein Rokkan entwickelt hat (Lipset und Rokkan 1967); die empirischen, auf Daten des World Values Survey, des Transatlantic Trends Survey und des WZB International Leaders Survey gestützten Beiträge des Bandes stärken die Hypothese des ,tectonic shift“: weg von nationalstaatlich eingehegten Verteilungskonflikten zwischen Kapital und Arbeit (bzw. auf der Parteienebene zwischen Konservativen und Sozialisten) hin zur Konfrontation zwischen Befürwortern und Gegnern diverser (ökonomischer, kultureller, politischer) Globalisierungsstrategien. Plakativ könnte man auch sagen: zwischen Verfechtern der offenen und geschlossenen Gesellschaft. ${ }^{1}$

Koopmans selbst zeigt freilich in einem weiteren Beitrag, wie viele Facetten beide „Lager“ aufweisen, und durchgängig wirkt die von den Autoren in Anspruch genommene Äquidistanz zu den beiden K-Polen problematisch. Kosmopoliten stehen, auch wo sie ein materielles Interesse an offenen Grenzen haben und in Distanz zu Unterschichten stehen, in einem objektiveren Verhältnis zur faktischen Externalisierung nationaler und lokaler Angelegenheiten, den Globalisierungswellen seit Jahrhunderten mit sich gebracht haben (Lessenich 2016), während Kommunitäre eine Begrenzung von Solidarität auf ,ihresgleichen“ nur noch schwer rechtfertigen können und das Risiko laufen, in ethno-nationalistische Positionen jenseits der liberalen Demokratie abzudriften. Schon Lipset hat 1970 mit Earl Raab die These aufgestellt, jede Verschiebung der Konfliktlinien ziehe einen Backlash nach sich, der soziale Gruppen, die real oder gefühlt ihrer Macht verlustig gehen, in den Rechtsextremismus treibt, faktisch vornehmlich weiße Männer (Lipset und Raab 1970). Eben dieser Trend bestätigt und verstärkt sich heute weltweit, kommunitäre Ontologien der Politik verlieren ihre Grundlage. Doch auch die Kosmopoliten müssen sich vom neoliberalen Globalisierungsdogma verabschieden und supranationale Regulierung, Umverteilung und Anerkennung befürworten. Die rhetorische und institutionelle Dominanz globalisierungsgeneigter Eliten hat nämlich eine kapitale Repräsentationslücke aufgerissen, und beide Strömungen verfehlen in ihrer Polarisierung das Angebot übergreifender politischer Projekte.

Die Generalhypothese des Bandes, die Merkel und Zürn im Abschlussbeitrag zuspitzen, wird damit nur unterstrichen: die Globalisierung hat eine neue Spaltungsund Konfliktlinie hervorgebracht, die einer sozialen Revolution gleichkommt. Das zeigt sich auf der normativen Ebene (in den Narrativen), in strukturellen Interessen-

\footnotetext{
1 Genauso hat Emmanuel Macron 2017 seinen Wahlkampf gegen Marine Le Pens Front National bestritten (vgl. Krouwel et al. 2019).
} 
gegensätzen und in einem organisatorisch-institutionellen Wandel etwa des Parteiensystems, der speziell die Sozialdemokratie erodieren ließ.

Der Band zeigt, wie spannend Politologie sein kann. Zu den offenen Forschungsfragen, die der Band aufwirft, gehört die Bedeutung der ökologischen Frage, die herkömmliche cleavages und Formationen erneut sprengen könnte, und die Rolle generationeller Oppositionen, die sich jüngst an dieser Überlebensfrage der Menschheit zu Wort gemeldet haben.

Funding Open Access funding provided by Projekt DEAL.

Open Access Dieser Artikel wird unter der Creative Commons Namensnennung 4.0 International Lizenz veröffentlicht, welche die Nutzung, Vervielfältigung, Bearbeitung, Verbreitung und Wiedergabe in jeglichem Medium und Format erlaubt, sofern Sie den/die ursprünglichen Autor(en) und die Quelle ordnungsgemäß nennen, einen Link zur Creative Commons Lizenz beifügen und angeben, ob Änderungen vorgenommen wurden.

Die in diesem Artikel enthaltenen Bilder und sonstiges Drittmaterial unterliegen ebenfalls der genannten Creative Commons Lizenz, sofern sich aus der Abbildungslegende nichts anderes ergibt. Sofern das betreffende Material nicht unter der genannten Creative Commons Lizenz steht und die betreffende Handlung nicht nach gesetzlichen Vorschriften erlaubt ist, ist für die oben aufgeführten Weiterverwendungen des Materials die Einwilligung des jeweiligen Rechteinhabers einzuholen.

Weitere Details zur Lizenz entnehmen Sie bitte der Lizenzinformation auf http://creativecommons.org/ licenses/by/4.0/deed.de.

\section{Literatur}

Goodhart, David. 2017. The road to somewhere: the populist revolt and the future of politics. London: C. Hurst \& Co.

Krouwel, André, Yordan Kutiyski, Oliver Philipp, und Arne Schildberg. 2019. “Macronism, Corbynism, ... huh?” Electoral Strategies of Progressive Political Parties in Europe. Bonn: Friedrich Ebert Stiftung. http://library.fes.de/pdf-files/id/ipa/15397.pdf.

Leggewie, Claus. 2017. Europa zuerst. Eine Unabhängigkeitserklärung. Berlin: Ullstein.

Leggewie, Claus. 2019. Jetzt! Opposition, Protest, Widerstand. Köln: Kiepenheuer \& Witsch.

Lessenich, Stephan. 2016. Neben uns die Sintflut: Die Externalisierungsgesellschaft und ihr Preis. München: Hanser.

Lipset, Seymour Martin, und Earl Raab. 1970. The Politics of Unreason: Right-Wing Extremism in America, 1790-1970. New York: Harper \& Row.

Lipset, Seymour Martin, und Stein Rokkan (Hrsg.). 1967. Party systems and voter alignments: crossnational perspectives. New York: The Free Press. 